\title{
Spatiotemporal dynamics of animal contests arise from effective forces between contestants
}

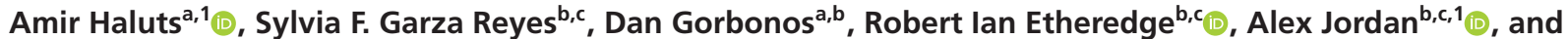 \\ Nir S. Gov ${ }^{\mathrm{a}, 1}$ (1) \\ ${ }^{a}$ Department of Chemical and Biological Physics, Weizmann Institute of Science, Rehovot 7610001, Israel; ${ }^{b}$ Department of Collective Behavior, Max Planck \\ Institute of Animal Behavior, Konstanz 78315, Germany; and ${ }^{\mathrm{C} C e n t r e ~ f o r ~ t h e ~ A d v a n c e d ~ S t u d y ~ o f ~ C o l l e c t i v e ~ B e h a v i o u r, ~ U n i v e r s i t y ~ o f ~ K o n s t a n z, ~ K o n s t a n z ~}$ \\ 78457, Germany
}

Edited by Robert Austin, Department of Physics, Princeton University, Princeton, NJ

Competition among animals for resources, notably food, territories, and mates, is ubiquitous at all scales of life. This competition is often resolved through contests among individuals, which are commonly understood according to their outcomes and in particular, how these outcomes depend on decision-making by the contestants. Because they are restricted to end-point predictions, these approaches cannot predict real-time or real-space dynamics of animal contest behavior. This limitation can be overcome by studying systems that feature typical contest behavior while being simple enough to track and model. Here, we propose to use such systems to construct a theoretical framework that describes real-time movements and behaviors of animal contestants. We study the spatiotemporal dynamics of contests in an orb-weaving spider, in which all the common elements of animal contests play out. The confined arena of the web, on which interactions are dominated by vibratory cues in a two-dimensional space, simplifies the analysis of interagent interactions. We ask whether these seemingly complex decision-makers can be modeled as interacting active particles responding only to effective forces of attraction and repulsion due to their interactions. By analyzing the emergent dynamics of "contestant particles," we provide mechanistic explanations for real-time dynamical aspects of animal contests, thereby explaining competitive advantages of larger competitors and demonstrating that complex decision-making need not be invoked in animal contests to achieve adaptive outcomes. Our results demonstrate that physics-based classification and modeling, in terms of effective rules of interaction, provide a powerful framework for understanding animal contest behaviors.

animal contests | spatiotemporal dynamics | physics-based modeling | size-related advantage

ompetition over resources, in which contestants act to obtain and defend resources such as food, territories, and mates, is an omnipresent feature of animal behavior. The contests themselves often involve resolution of complex trade-offs between risk and reward, even in relatively simple organisms (1-6). Consequently, models developed to describe animal contests and predict their outcomes have traditionally relied on the ability of contestants to perform nontrivial computations, such as assessing their own or their rival's resource holding potential, and to make cognitive decisions accordingly $(1,7-11)$. These game-theoretic models provide important insights into the nature of animal contests under different assessment strategies, but they remain difficult to validate and discriminate between $(12,13)$. This difficulty stems in part from the inherent challenge of verifying the cognitive decision-making paradigms at the basis of these models in behavioral experiments. Moreover, the most apparent and easily measurable aspects of animal contests-their spatiotemporal dynamics-are generally beyond the scope of existing models. Modern tracking capabilities dramatically increased the resolution at which the dynamics of animal behavior can be investigated (14). These capabilities facilitate quantitative behavioral experiments across systems and scales (15) and motivate analytical and modeling approaches in the study of animal contests.
Contests in many species share unifying elements, which we propose to analyze in terms of their effects on the motion of the contestants. One common aspect of contests is that a spatially localized resource is detected and approached by potential rivals based on the perceived resource value $(1,16-18)$. The resource acts as an attractor that drives the contestants closer to one another until conflict is inevitable. After the contestants reach the contest distance, their dynamics are governed by behavioral elements of aggressive conflicts $(7,19)$, which have been previously described as escalating stages of "sequential assessment" $(9,20$, 21). These manifest in space as regimes of effective repulsion or attraction, where each contestant tends to move away from or toward its rival as a consequence of behaviors such as displays, fights, and retreats. Motivated by these general characteristics, in this work we develop a theoretical framework that describes the dynamics of contestants as responses to effective forces, which arise due to their interactions with each other and with a resource. From the resulting equations of motion, we obtain the spatiotemporal dynamics of the contestants. This approach has the potential to greatly improve the analysis of contests as these dynamics are directly observable from the behavior and can be analyzed without invoking cognitive processes that cannot be directly verified from the collected data.

Here, we study the dynamics of animal contests empirically and theoretically in a natural setup-the bounded arena of the Trichonephila female spider web, upon which rival males that can

\section{Significance}

To obtain and defend resources, animals often participate in contests that involve complex trade-offs between risk and reward. Although it stands to reason to invoke cognitive decision-making schemes to analyze and model these contests, such cognitive schemes are hard to verify in behavioral experiments, and they do not address a directly observable aspect of contests-the motion of contestants in space. We study the dynamics of contests in an orb-weaving spider, where males compete for mating opportunities in the bounded arena of the female's web. We show that physical rules of interaction, which amount to attraction and repulsion between the contestants and govern their motion, can account for the real-time dynamics of animal contests.

Author contributions: A.H., S.F.G.R., A.J., and N.S.G. designed research; A.H., S.F.G.R., and D.G. performed research; A.H., R.I.E., and N.S.G. analyzed data; and A.H., A.J., and N.S.G. wrote the paper.

The authors declare no competing interest.

${ }^{1}$ To whom correspondence may be addressed. Email: amir.haluts@weizmann.ac.il, ajordan@ab.mpg.de, or nir.gov@weizmann.ac.il. 
vary greatly in size $(22,23)$ compete among themselves for an opportunity to mate with the much larger and potentially cannibalistic female (24-27). The two-dimensional (2D) and bounded geometry of the web allows us to track the motion of these agents with great detail in naturalistic field experiments. This system features many of the elements of contest behavior in other animal taxa, particularly with respect to intermale competition (28). These males act to gain access to a spatially localized resourcethe position closest to the female-and restrict the access of rivals through competitive interactions $(24,29)$. A simplifying element is that, as in other orb-weaving spiders, the interactions in this system are dominated by a single modality of vibratory cues (30-32). These features allow us to construct an empirically motivated theoretical model, which describes the dynamics of agents that compete over a resource. The model is framed in terms of effective potentials that give rise to effective interaction forces between the agents, which in turn, govern their spatiotemporal dynamics.

We use our model to test the hypothesis that the apparently complex processes of competitive behavior in these spiders can be accounted for by the dynamics of interacting active particles, which respond only to effective forces of attraction and repulsion. This approach assumes no higher psychological processes on the part of the contestants [i.e., Morgan's canon (33)] and therefore, presents a useful null hypothesis in the study of animal contests, against which more complex behavioral interpretations can be evaluated. We further examine contests between differentsized rivals by mapping the sizes of the contestants to their effective interaction potentials. Size is commonly associated with increased competitive ability in animal contests (13), and positive selection on body size through enhanced competitive ability has been suggested in numerous taxa (34-38). This effect may be augmented at high male densities, where greater contact rate among contestants can magnify competitive size advantages, increasing reproductive skew within the male population (39). The model predicts a qualitative change in contest dynamics, from symmetric bounded tussles to directional chases, as the size difference between the contestants increases. We provide experimental evidence for this trend and relate it to the predicted change in contest duration with size asymmetry. Our theoretical findings suggest that large males gain an advantage, especially at high male density, as a direct result of their effective interaction potentials, which make them better than smaller males at avoiding short-range interactions and long-lasting contests. These results demonstrate the importance of spatiotemporal data and the usefulness of physics-based modeling in understanding the dynamics of animal contests.

\section{Results}

We describe an empirical and theoretical exploration of the dynamics of male contestants in the orb-weaving spider Trichonephila clavipes. These males migrate at maturity to the webs of females in search of a mate (24). After a male has entered a female's web, it relies primarily on vibratory information to interact with the female and with rival males $(32,40)$. The female's web serves as a natural interaction arena for male contestants, where the dynamics of competition over a well-defined and localized resource can be studied in great detail. We conducted controlled experiments in this system (Materials and Methods) in order to tease apart the effective physical rules that govern male-female and male-male interactions on the web. We rely on these rules to construct our theoretical model for the behavior of the males and use it to investigate intermale competition in simulations.

Model for Male-Female Interactions. The influence of the female and the architecture of its web on the dynamics of males can be thought of as the resource landscape that brings males into competitive encounters. Modeling the effect of this landscape on the behavior of male contestants is the starting point for our theoretical description. Although the typical trajectories along which males travel toward the female may be specific to our studied web environment, the property of resource patches to bring contestants into contest distance is generic. Furthermore, the following methods by which we construct and optimize our model for the resource landscape are applicable to localized resources in general.

During most of the day, the female assumes a static, downfacing position at a dedicated location in the structural center ("hub") of the orb and waits for prey to get caught $(41,42)$. Females are highly reactive toward vibratory signals that they interpret as potential prey captures, and this attitude is most prominent when the source of vibrations is within the main prey capture zone of the web, which occupies a wide sector in front of the hub-dwelling female $(43,44)$ ("frontal sector") (Fig. $1 A)$. $T$. clavipes males may have evolved to utilize the well-defined and conserved architecture of female webs to navigate their way toward the female and minimize the premating risk of predation.

In agreement with ref. 43, we observed that males tend to avoid the frontal sector and approach the female from behind, where it is least reactive. In single-male experiments (Materials and Methods and SI Appendix, Figs. S1 and S2), we demonstrate that this behavior leads to effective ordered "flow" of males around the female and toward a desired spot within the hub (Fig. $1 B$ and $C$ ), where a male would wait for an opportunity to copulate. In the absence of rival males, this flow is associated with the influence of the female and the architecture of its web. Since the position of the female is essentially fixed in the timescales of interest, its influence can in turn be treated as an effective "female potential," $V_{\mathrm{fem}}$. This effective potential describes the localized resource attractor of this system and its averaged effect on approaching males.

As the males are evidently attracted to the female but avoid the frontal sector (Fig. $1 B$ and $C$ ), it is natural to construct $V_{\text {fem }}$ as a combination of two components-isotropic attraction and anisotropic repulsion. The isotropic attraction is associated with the movement of males toward the female from the edges of its web and is, therefore, long range with respect to the dimensions of the system. The anisotropic repulsion expresses the observed avoidance of the frontal sector by approaching males that are aiming to evade detection by the female.

The combination of isotropic and anisotropic contributions to the effective female potential, which acts in the 2D surface of the web, suggests that we write $V_{\text {fem }}$ as the leading terms of a 2D multipole expansion (45). The long-range (monopole) term corresponds to the isotropic attraction, while the shorterrange (dipole-like) term corresponds to the anisotropic repulsion (SI Appendix, Fig. S3). Namely, we propose to describe $V_{\text {fem }}$ by the potential

$$
V_{\mathrm{fem}}(r, \theta)=\alpha \ln r+\beta \frac{C+\cos \theta}{r},
$$

where $(r, \theta)$ is the position of the male in the female-centered coordinate system, as defined in Fig. $1 A$, and $\alpha, \beta>0$ are parameters that set the relative contribution of each term. The constant $C \geq 1$ in the numerator of the dipole-like term imposes that this signal is never attractive. The potential of Eq. 1 is visually represented in SI Appendix, Fig. S3. The gradient of Eq. 1 generates a flow field with two fixed points-one of which is globally stable and the other of which is an unstable saddle (SI Appendix, Fig. S3 and section S1). These properties of $V_{\text {fem }}$ can be directly related to their experimental analogs. The globally stable fixed point is associated with the location of the hub, and the unstable saddle point is associated with the boundary of the frontal sector directly in front of the down-facing female (Fig. $1 A$ ), as they are perceived by males. 
A

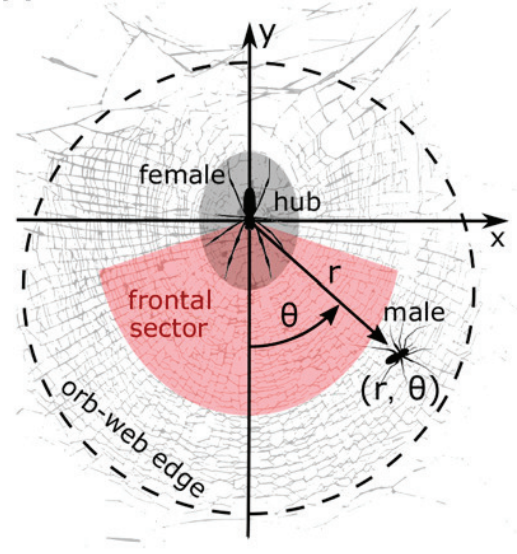

B

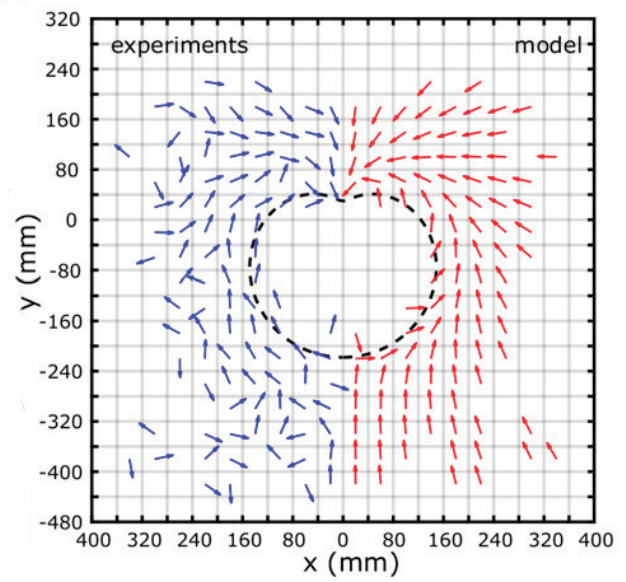

C

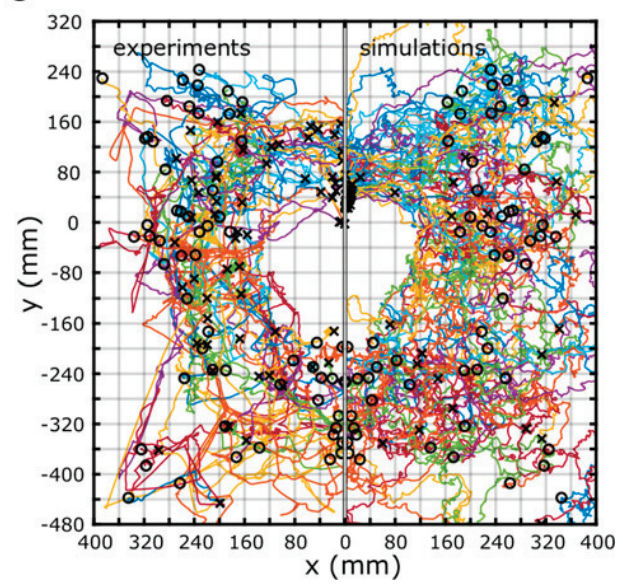

Fig. 1. Single-male dynamics. (A) Female-centered coordinate system. The head of the static, down-facing female is taken as the origin. The position of a male in this reference frame is shown in polar coordinates. Colored areas roughly illustrate the location and shape of the hub and the frontal sector. The dashed circle approximates the rim of an adult female's orb web, within which real males can essentially travel continuously. ( $B$ ) Flow fields comparison. The normalized gradient field (director field) of the optimized female potential (Eq. 1 with $\beta / \alpha=94, C=1.32$ ) is shown alongside its experimental analog (calculated directly from the experimental trajectories of $C$ ) to allow for a visual comparison. Each director indicates the mean local direction of trajectories that pass through its bin, bordered by the grid square. The dashed contour indicates where the radial partial derivative of the female potential is zero $\left(\partial V_{\mathrm{fem}} / \partial r=0\right)$. (C) Single-male trajectories comparison $(N=59)$. Each experimental trajectory was obtained from an independent experiment, in which a single male was placed at some initial position and left to move freely in the presence of the female for about 10 min. The simulated single-male trajectories were produced for the same initial positions and durations as the experimental ones. Initial and final positions are marked by circles and $x$ 's, respectively. In $B$ and $C$, the data are "folded" along the axis of symmetry (the $y$ axis) onto one half-plane. In $B$, directors in spatially equivalent bins were further averaged postfolding. SI Appendix, Fig. S2 shows the unfolded experimental trajectories.

Since the motion of males on the web is mostly slow and characterized by frequent pauses, we neglect the effects of inertia and take the local velocity of isolated males to be directly proportional to the local gradient of $V_{\text {fem }}\left(\nabla V_{\text {fem }}\right)$. By comparing the normalized flow field (director field) of $V_{\text {fem }}$, given by $-\nabla V_{\text {fem }} /\left|\nabla V_{\text {fem }}\right|$, with its experimental analog obtained from single-male trajectories (Fig. $1 B$ ), we optimized the parameters of $V_{\text {fem }}$ such that the directional discrepancy between the two flow fields is minimal (SI Appendix, Fig. S4 and sections S2 and S3).

Compared with the deterministic flow field generated by $-\nabla V_{\text {fem }}$, experimental single-male trajectories are characterized by positional noise and notable directional persistence, as depicted qualitatively in Fig. $1 C$. To incorporate these characteristics into our theoretical framework, we treat the males as active Brownian particles (46) in an external effective potential (as in ref. 47) (SI Appendix, Fig. S5 and sections S4 and S5). A comparison between experimental and simulated male trajectories is shown in Fig. $1 C$ as a visual validation of the model thus far. This validation relies on the already optimized female potential and therefore, establishes the effective physics that govern the motion of single males on the female's web. Note that our simulations successfully describe the overall shape and spatial distribution of full trajectories, while the temporal dynamics of real males are affected by sporadic pauses that are not explicitly included in the model.

Model for Male-Male Interactions. By constructing and optimizing $V_{\text {fem }}$, we obtained the resource landscape on which male-male interactions take place. We now describe the physical implications of male-male interactions in this system and complete our theoretical description by constructing the effective interaction potentials of the males. Although the precise functional form and parameters of these interaction potentials are specific to our studied system, the behavioral elements on which they are based and the methods used to construct them are much more general and therefore, applicable to many other systems. Namely, in the following we analyze the interactions in terms of distancedependent behavioral regimes, in which the tendency of each contestant to move away from or toward its rival is interpreted as effective repulsion or attraction between the contestants.

On the female's web, males compete with rivals to obtain and retain the desired hub position closest to the female. Previous studies have described the stages of escalation in contest behavior as the distance between the rivals decreases $(24,43,48,49)$. When two males first become aware of one another, they try to intimidate the rival by actively jerking the web to generate vibrations (43). If these long-range vibratory threats did not yield the desired response, a persistent aggressor will move directly toward its rival, which either retreats or becomes engaged with the aggressor and prepares for a fight (48). Finally, if both males persist, they will come into contact and grapple (49) in an effort to forcefully chase their opponent away (24). Although males themselves are unlikely to injure one another in these fights, conflict between males creates vibrational disturbances on the web that can lead to attack by the larger female, so male-male competition is inherently risky (50). We observed that males keep employing vibratory threats as they get closer to each other, probably as a means of resolving the encounter without the ultimate escalation to grappling.

To understand the dynamics of intermale competition, we conducted controlled two-male experiments, in which we allowed two males to interact in a female's web (Fig. $2 A$ and $B$, Materials and Methods, and Movie S1). From these experiments, we extracted the averaged intermale relative velocity as a function of the intermale distance $d$ ("intermale velocity profile") (Fig. 2C). This measure relates the behavior of interacting males to its physical implications, namely the direction and speed at which they tend to move with respect to each other as they get closer or farther apart. In agreement with the behavioral observations, the intermale velocity profile reveals three distinct interaction regimes (Fig. 2C). We attribute positive intermale velocity (a tendency to increase $d$ ) to effective repulsion between the males and negative intermale velocity (a tendency to decrease $d$ ) to effective attraction, which is interpreted, in accordance with observations, as escalation in aggression. In analyzing Fig. $2 C$, one should consider the residual attraction of both males to the female, which shifts their relative velocity toward more negative 


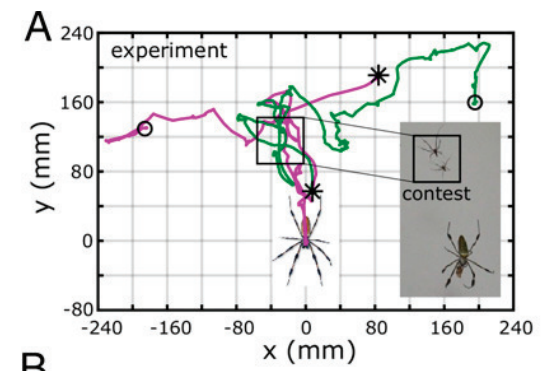

B
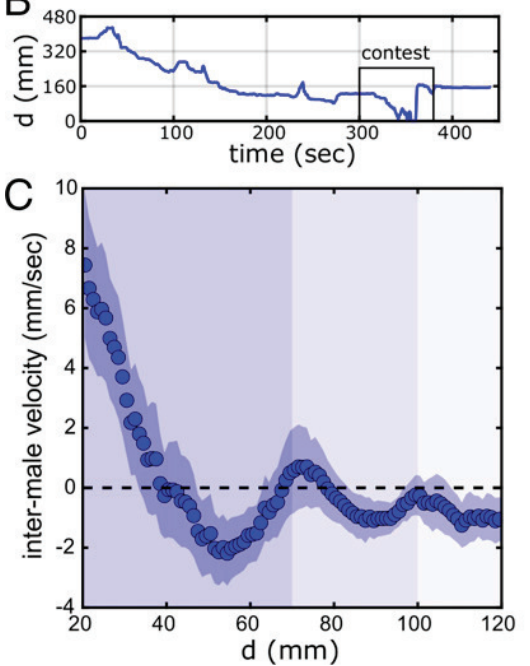

$\mathrm{D}$
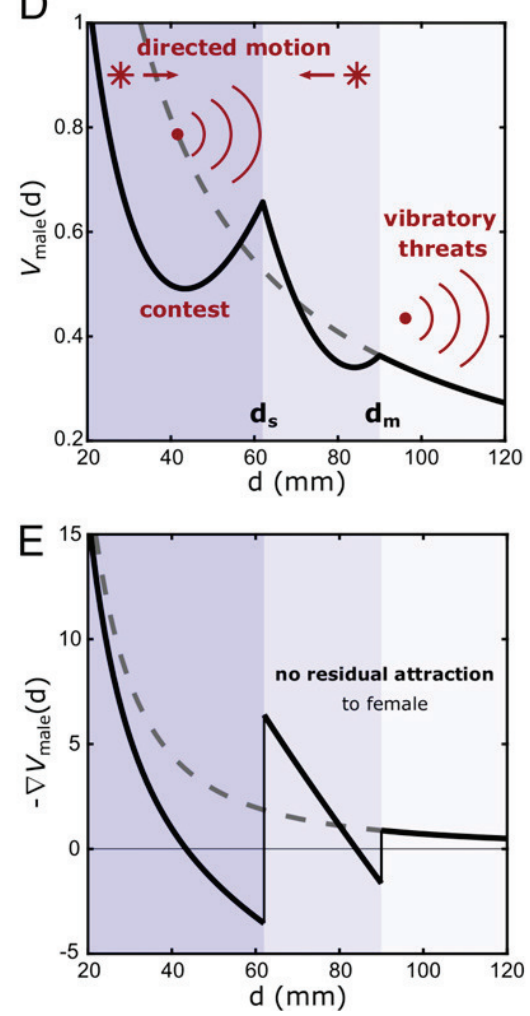

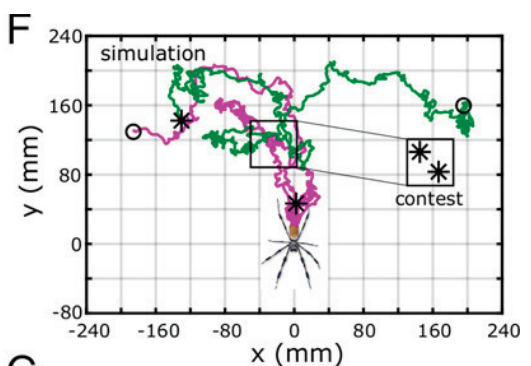

G
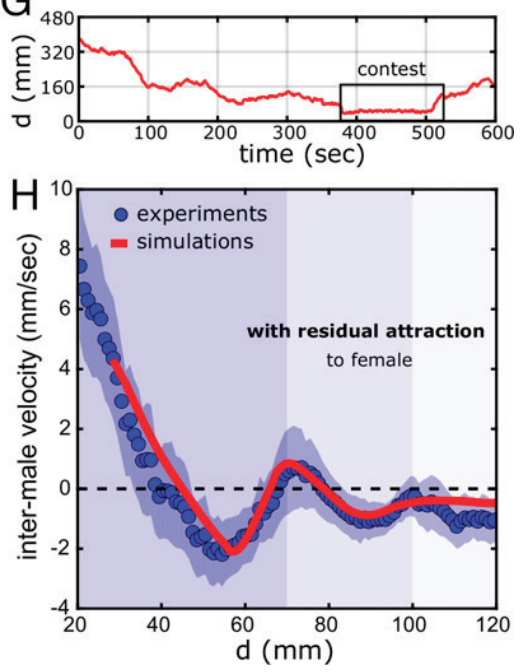

Fig. 2. Two-male dynamics. (A) Example of male trajectories from a two-male experiment. In these experiments, two males were placed in a female's web near the rim of the orb's upper part and left to freely move and interact. This particular instance features a contest between the males (a snapshot is in Inset), which resulted in a swap for the hub position. Initial and final positions are marked by circles and asterisks, respectively. Movie $\mathrm{S} 1$ shows the tracked male positions during this experiment. $(B)$ The distance between the males throughout the experiment in $A$. (C) Averaged intermale (relative) velocity as a function of intermale distance $d(N=29)$. Error bars show SEM. $(D)$ The effective male potential $V_{\text {male }}($ Eq. 2$)$, with the parameter values $\delta=32.7, k=4 \times 10^{-4}$,

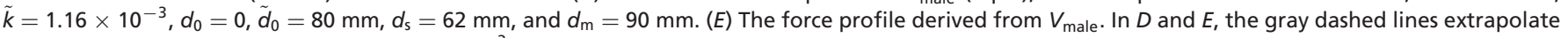
the vibration-based repulsive term $\left(\delta / d\right.$ and $\delta / d^{2}$, respectively). $(F)$ An example of male trajectories from a two-male simulation, with initial positions as in $A$. $(G)$ The distance between the males throughout the simulation in $F$. $(H)$ Intermale velocity profiles comparison. The red profile was obtained from simulations as in $F(N=2,000)$, with parameter values as in $D$. Velocities were calculated with a time window of $2 \mathrm{~s}$ (50 video frames or simulation steps). In $C-E$ and $H$, the different shaded bands indicate the three distance-dependent regimes of intermale interaction. The bands in $D$ and $E$ correspond to the optimized values of $d_{\mathrm{s}}$ and $d_{\mathrm{m}}$, and the bands in $C$ and $H$ are visual estimates. In $C$ and $H$, a moving averaging window of length 11 mm was used for smoothing.

values. This effect is most prominent when the males are far apart and not strongly engaged with each other (as in the right-most region of Fig. $2 C$ ), so that the attraction toward the female is of the order of the intermale effective force.

Following these considerations, we can directly relate the intermale velocity profile to an effective "male potential," $V_{\text {male }}$ (Fig. 2D). This effective potential captures the averaged effect of an isolated male-male interaction on the dynamics of the males. Under the premise that inertial effects can be neglected, the local intermale velocity between two isolated males is proportional to the local gradient of $V_{\text {male }}$. To construct $V_{\text {male }}$, we assume that the effective intermale forces, which govern the motion of males with respect to each other, depend only on the intermale distance $d$. This assumption is valid on average, as males rotate to face their opponents when they become engaged with them.

The most basic contribution to $V_{\text {male }}$ is vibration-based repulsion, which arises from the vibratory threats described above. Since males initially get closer to each other due to their attraction to the female, we expect the vibratory threats to be of shorter range than the attractive logarithmic term of $V_{\text {fem. }}$. Hence, we propose that the vibration-based repulsion decays as $1 / d$, much like the dipole-like repulsive term of $V_{\text {fem }}($ recall Eq. 1). When $d$ decreases beyond a certain average threshold $(d<100 \mathrm{~mm}$ in Fig. $2 C$ ), the aggression escalates through active directed motion of the males toward each other. As the males tend to decrease $d$ when engaged in this type of interaction, it amounts to effective attraction. Fig. $2 C$ suggests that this behavior should be further dissected into two distinct behavioral regimes, separated by a short-range "barrier" (at $d \approx 70 \mathrm{~mm}$ ). We obs3erve that reaching the short-range regime $(d<70 \mathrm{~mm})$ often entails commitment to grappling, and therefore, it can be regarded as the "contest regime" for these male contestants. Since these regimes are characterized by relatively short-range linear motion, we model their contributions to $V_{\text {male }}$ using spring-like terms of the form $(d-$ $\left.d_{0}\right)^{2}$, where $d_{0}$ is an equilibrium distance for the directed motion. These spring-like terms complement the basic vibration-based repulsive term of $V_{\text {male }}$ when the intermale distance decreases below $d \approx 100 \mathrm{~mm}$ (Fig. $2 D$ ). We thereby describe $V_{\text {male }}$ by the following piecewise potential:

$$
V_{\text {male }}(d)=\left\{\begin{array}{lr}
\frac{\delta}{d}+\frac{k}{2}\left(d-d_{0}\right)^{2}+c_{0}, & d \leq d_{\mathrm{s}} \\
\frac{\delta}{d}+\frac{\tilde{k}}{2}\left(d-\tilde{d}_{0}\right)^{2}+\tilde{c}_{0}, & d_{\mathrm{s}}<d \leq d_{\mathrm{m}}, \\
\frac{\delta}{d}, & d_{\mathrm{m}}<d
\end{array}\right.
$$

where $\delta>0$ is the strength of the vibration-based repulsion; $k$ and $\tilde{k}$ are the effective spring constants for the short-range and medium-range directed motion, respectively; $d_{0}$ and $\tilde{d}_{0}$ are the corresponding spring equilibrium distances for these two regimes; $d_{\mathrm{s}} \approx 70 \mathrm{~mm}$ and $d_{\mathrm{m}} \approx 100 \mathrm{~mm}$ are the short-range and 
medium-range regime onsets, respectively; and $c_{0}$ and $\tilde{c}_{0}$ ensure the continuity of the potential in these regime onsets. Note that the parameters of Eq. 2 are tightly constrained by the shape of the experimental intermale velocity profile (Fig. $2 C$ ). The potential of Eq. 2 and the effective force profile derived from it are plotted in Fig. $2 D$ and $E$.

We further note that (according to our observations) when two males take part in a short-range interaction $\left(d \leq d_{\mathrm{s}}\right)$, they are completely occupied with each other and would not resume their approach toward the female until the encounter is resolved. This means that the attraction to the female is effectively "turned off" from the perspective of the interacting males when $d \leq d_{\mathrm{s}}$. To incorporate this behavioral feature in our model, we impose that the attractive term of $V_{\mathrm{fem}}$ is active only when the males are not engaged in a short-range interaction, so that $V_{\text {fem }}$ is given only by its repulsive dipole-like term if $d \leq d_{\mathrm{s}}$ and by Eq. 1 in its entirety if the males are farther apart. Therefore, the dynamics of malemale contests, which we define to take place when $d \leq d_{\mathrm{s}}$, are not strongly dependent on the shape of the female's potential landscape.

For the full theoretical description of a male in a female's web, we consider its interactions with the female and with all the other male rivals. The dynamics of male $i$ at position $\mathbf{r}_{i}=\left(r_{i}, \theta_{i}\right)$ are governed by its interaction with the effective female potential, given by $V_{\mathrm{fem} \rightarrow i}\left(\mathbf{r}_{i}\right)$, and by its interactions with the effective male potential of any other male $j$ at position $\mathbf{r}_{j}=\left(r_{j}, \theta_{j}\right)$, given by $V_{\text {male } j \rightarrow i}\left(\left|\mathbf{r}_{i}-\mathbf{r}_{j}\right|\right)$. The arrows emphasize the directionality of these interactions. Assuming linear superposition of pairwise interactions, male $i$ is, therefore, subjected to a total effective potential, $V_{\text {tot } \rightarrow i}$, that is given by the sum of individual effective potentials:

$V_{\text {tot } \rightarrow i}\left(\mathbf{r}_{i},\left\{\mathbf{r}_{j}\right\}\right)=V_{\text {fem } \rightarrow i}\left(\mathbf{r}_{i}\right)+\sum_{j \neq i} V_{\text {male } j \rightarrow i}\left(\left|\mathbf{r}_{i}-\mathbf{r}_{j}\right|\right)$.

We used Eq. 3 to simulate the two-male case (Fig. $2 F$ and $G$ ). By adjusting the parameters of Eq. 2, we obtained from these simulations an intermale velocity profile that is in good agreement with the experimental profile (Fig. $2 H$ ). This agreement establishes the effective physics of intermale competition in a female's web. In SI Appendix, section S6, we omit the mediumrange spring-like regime $\left(d_{\mathrm{s}}<d \leq d_{\mathrm{m}}\right)$ from Eq. 2 and repeat the comparison of Fig. $2 H$ (SI Appendix, Fig. S6). The results emphasize that this regime is necessary to reproduce the shape of the experimental intermale velocity profile. For all of the simulations in this work, we represent the edge of the orb web as a circle (Fig. $1 A$ and SI Appendix, Fig. S7). The simulated males are considered as interacting (according to Eqs. $\mathbf{2}$ and $\mathbf{3}$ ) only when they are found within the orb-defining circle, and the trajectory sections used to analyze simulation results were only those that remained within this boundary.

Interactions between Males of Different Sizes. For contests between males of similar sizes, we have demonstrated that the dynamics of our model's "contestant particles" are in quantitative agreement with real male interactions. However, sexually mature males of the genus Trichonephila exhibit an exceptionally high variation of sizes $(22,23)$. This poses a long-standing challenge in understanding selection on male body size in these spiders, as males that differ substantially in size are often found to coincide and compete for the same females. To study the role of size in intermale competition, we need to represent males of different sizes in our model. In the following, we associate the optimized male potential of Fig. $2 H$ with the interaction potentials of two "reference" (averaged) males and explore the size-related deviations from this reference interaction.

When two different-sized males $i$ and $j$ interact, their respective interaction potentials, $V_{\text {male } i \rightarrow j}$ and $V_{\text {male } j \rightarrow i}$, should exhibit the broken symmetry of their interaction. The first contribution to this asymmetry arises since larger males can generate and transmit stronger vibrations on the web surface. In accordance with the dominance of vibratory signals in the interactions of these spiders, we assume that the only input a male has about the size of its rival is the strength of its transmitted vibrations. Importantly, we assume that a male does not directly assess its rival's fighting ability $(9,20,51)$. Furthermore, previous studies of contest behavior in several species of spiders suggest that spiders assess their own state to decide whether to escalate the conflict or retract from it, in support of the "self-assessment" paradigm (52-55). We account for these effects by varying the strength of the vibration-based repulsive term and of the short-range springlike attractive term of $V_{\text {male }}$, given by the values of $\delta$ and $k$ in Eq. 2. For the interaction potential of male $i$, which is felt by its rival $j\left(V_{\text {male } i \rightarrow j}\right)$, these parameters are now denoted $\delta_{i}$ and $k_{i}$. Note that $\delta_{i}$ is directly associated with the size of male $i$, while $k_{i}$ is associated with the size-related behavior of the rival male $j$, as this parameter reflects how "willing" is $j$ to escalate the interaction with $i$ and therefore, captures the self-assessment of $j$. For simplicity, we assume that both $\delta_{i}$ and $k_{i}$ vary linearly with the size (weight) of the males. That is, if $m_{i}$ and $m_{j}$ denote these respective sizes, then $\delta_{i}=\delta_{0} m_{i}$ and $k_{i}=k_{0} m_{j}$, where $\delta_{0}$ and $k_{0}$ are independent of size. For the reference interaction potential of Fig. $2 H$, we set the theoretical reference "sizes" to $m_{\text {ref }}=1$, such that the values of $\delta_{0}$ and $k_{0}$ are those used for $\delta$ and $k$ in Fig. 2.

The symmetry breaking in the interaction of different-sized males has a measurable effect on contest dynamics that becomes more prominent if the size difference is large. When a male $i$ interacts with a larger male $j$, the larger male exerts a stronger effective repulsive force on the smaller male than the smaller male does on the larger male since $\delta_{j}>\delta_{i}$. Moreover, the larger male feels a stronger effective attraction toward the smaller male in the short-range contest regime $\left(d \leq d_{\mathrm{s}}\right)$ since $k_{i}>k_{j}$. This asymmetry in the effective intermale forces, which are exerted in opposite directions along the intermale axis, gives rise to "chase" dynamics at small $d$, where large males tend to chase smaller males away. We can measure the chase in pairs of interacting males by considering the mean correlation between the velocity of the pair's center of mass, $\hat{\mathbf{v}}_{\mathrm{cm}}$, and the intermale direction vector, $\hat{\mathbf{r}}_{i j}$, as defined in Fig. $3 A$. This "chase correlator," denoted by $\left\langle\hat{\mathbf{v}}_{\mathrm{cm}} \cdot \hat{\mathbf{r}}_{i j}\right\rangle$, evaluates the extent of asymmetry in the interaction. It is positive and large if significant chase dynamics take place, as predicted for the case of large size difference, and is nearly zero for the symmetric contests between males of similar sizes (Fig. $3 B$ and Movies S2 and S3 show simulations of these two cases). We further find that the average speed of males on the web weakly increases with their size (SI Appendix, Fig. S8). As this increase in speed may contribute to the competitive advantage of size, we account for this trend in our model (SI Appendix, section S7).

In Fig. $3 B$ and $C$, we calculated the mean chase correlator in two-male simulations and experiments for the case of large size difference and for the case of similar sizes. The predicted chase in the case of large size difference, as well as the nearly zero correlation for the symmetric case, clearly appears in both simulations and experiments. Example trajectories that demonstrate these two types of dynamics are shown in Fig. $3 D-G$ (Movies S2-S5 correspond to these trajectories). This comparison validates our theoretical treatment for males of different sizes and motivates us to use our model to study realistic scenarios in intermale competition, in which size may play an important role.

Animal contests are often characterized according to the dependence of their duration on the contestants' sizes and size difference (13). In our model, a contest is initiated when two males reach a separation $d$ that is shorter than $d_{\mathrm{s}}$ (Fig. 2D). The contest terminates either when $d$ increases beyond $d_{\mathrm{s}}$ (as in Fig. $3 D$ and Movie S2) or when one of the males is 

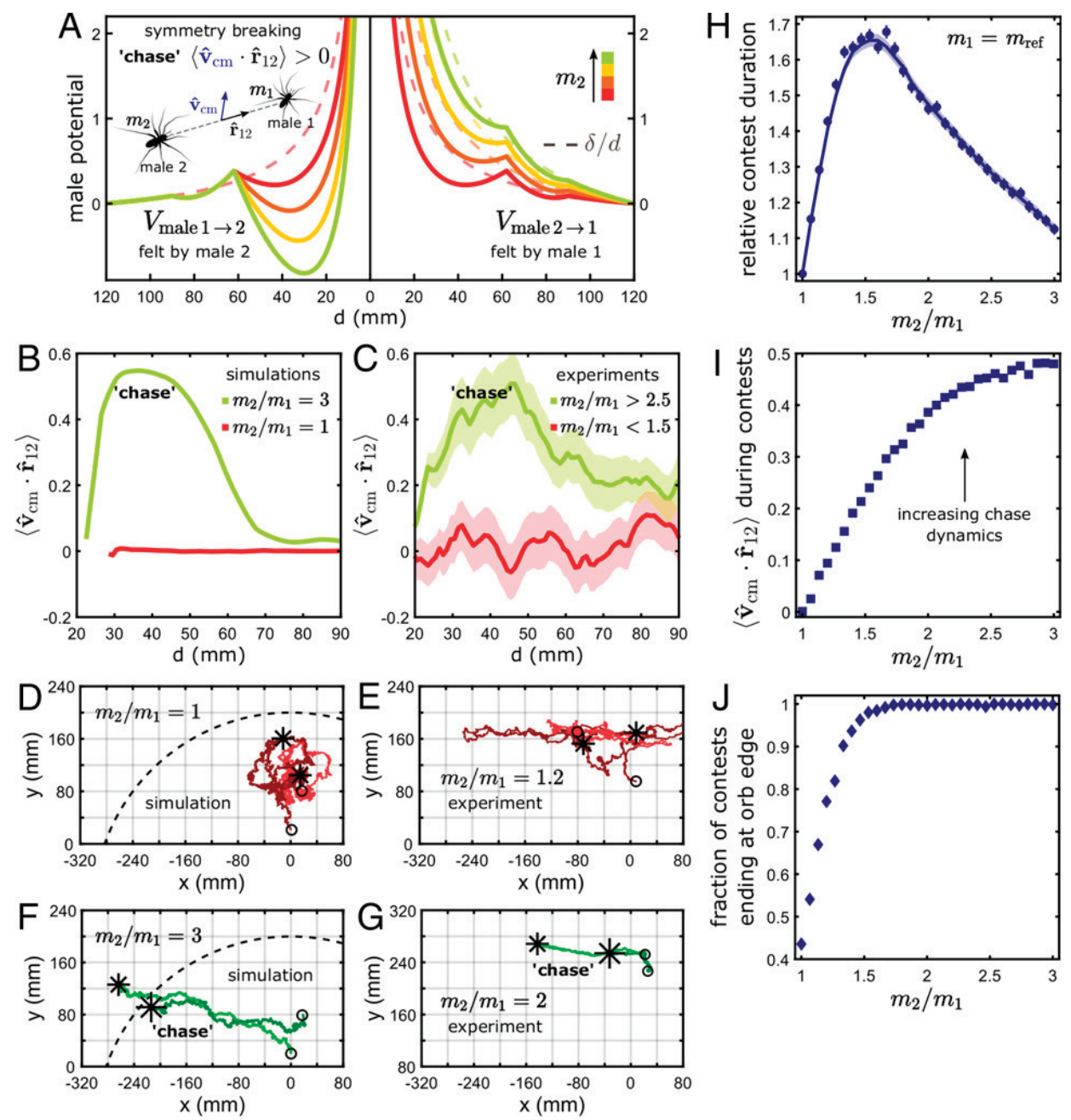

Fig. 3. Asymmetric contests. $(A)$ When two different-sized males (male $2>$ male 1 ) interact, their effective potentials exhibit the broken symmetry of their interaction. The strength of the vibration-based repulsive term of the potential exerted by the larger male on the smaller male $\left(\delta_{2}\right.$ in $\left.V_{\text {male }} \rightarrow 1\right)$ increases with the size of male $2\left(\delta_{2} \propto m_{2}\right)$, as shown in Right. Due to self-assessment, larger males have a stronger tendency to escalate the conflict, and this is manifested by an increased strength of the short-range attractive term of the potential exerted by the smaller male on the larger male $\left(k_{1} \propto m_{2}\right.$ in $\left.V_{\text {male } 1 \rightarrow 2}\right)$, as evident by the deepening potential well in Left. Due to these effects, the smaller male feels a more repulsive potential with a shallower minimum. This gives rise to chase dynamics at small intermale distance $d$. The chase is manifested by positive mean correlation between the velocity of the males' center of mass $\hat{\mathbf{v}}_{\mathrm{cm}}$ and the intermale direction vector $\hat{\mathbf{r}}_{12}$, denoted by $\left\langle\hat{\mathbf{v}}_{\mathrm{cm}} \cdot \hat{\mathbf{r}}_{12}\right\rangle$ (the chase correlator). (B) Simulated profile of the chase correlator. Symmetric case: $m_{2} / m_{1}=1$, with $m_{1}=m_{\text {ref }}=1$. Asymmetric case (large size ratio): $m_{2} / m_{1}=3$, with $m_{1}=m_{\text {ref }} / 2(N=2,000$ simulations). (C) Experimental profile of the chase correlator. Symmetric case (mean \pm SD): $m_{2} / m_{1}=1.2( \pm 0.1)$, with $m_{1}=12( \pm 3) \mathrm{mg}$ and $m_{2}=14( \pm 4) \mathrm{mg}(N=19$ experiments). Asymmetric case: $m_{2} / m_{1}=2.9( \pm 0.3)$, with $m_{1}=4.4( \pm 0.9) \mathrm{mg}$ and $m_{2}=13( \pm 3) \mathrm{mg}\left(N=10\right.$ experiments). In $C, m_{i}$ denotes the weights of the males. Error bars show SEM. In $B$ and $C$, a moving averaging window of length $11 \mathrm{~mm}$ was used for smoothing. $(D-G)$ Examples of contest trajectories from simulations and experiments for the interaction of size-matched males $(D$ and $E$ ) and for males that differ substantially in size $(F$ and $G)$. Circles and asterisks denote the initiation and termination of the contests, respectively. In $F$ and $G$, the chase dynamics are evident. In $D$ and $F$, the dashed lines denote a portion of the orb-defining circle, as defined in SI Appendix, Fig. S7. $(H)$ Mean contest duration as a function of male size ratio in simulations $(N=2,000)$. The trend corresponds to holding the size of the smaller male 1 constant $\left(m_{1}=m_{\text {ref }}\right)$ while increasing the size of male 2 . The line is a five-points moving average. Error bars show SEM. (I) Mean value of the chase correlator during the contests in $H$. $(J)$ The fraction of contests in $H$ ending when one male is driven to the edge of the orb (as in F). Only in the case of two size-matched males of the reference size does a significant fraction of the contests end due to spontaneous detachment within the orb-defining circle (as in $D$ ).

driven outside of the orb edge (as in Fig. $3 F$ and Movie S3). In Fig. $3 H$, we plot the mean contest duration as a function of male size ratio as calculated from simulations. When the size of the smaller (reference) male ("male 1") is held constant, the contest duration initially increases with the size of the larger male ("male 2"). This trend is characteristic of contests resolved through self-assessment (13) and indicates that our implementation of self-assessment in terms of size-dependent variations in the effective intermale attractive potential captures the effect of behavioral self-assessment on contest duration. However, the trend is reversed above a certain size ratio, and this is not expected for pure self-assessment. The decrease in contest duration for a large size ratio is a result of increasing chase dynamics (Fig. 3I), which lead to contests that typically terminate when the smaller male is driven out of the orb (Fig. $3 J$ ). The duration of these chase interactions is determined by the speed of the larger male (the chaser), which weakly increases with its size (SI Appendix, Fig. S8). Note, however, that chase dynamics alone are sufficient to reverse the trend of increasing contest duration with size ratio in this spatially bounded system (SI Appendix, Fig. S9). In SI Appendix, Fig. S10 and section S8, we consider a simpler model for the size-dependent variations of intermale interactions that does not include variations in the effective attractive components of the male potentials (does not include self-assessment). In this simpler model, only the vibration-based repulsive components of the potentials depend 
on size. While this model is sufficient to reproduce the chase dynamics of asymmetric contests, it also predicts that contests between large males are significantly shorter than contests between small males, which is inconsistent with our observations. This supports the assumption that some form of self-assessment is employed in these contests.

The Effects of Local Male Density on Large-Male Advantage. It is observed that local male density, defined as the number of males occupying a single female's web, is highly variable in wild populations of T. clavipes. A recent study related this observation to the puzzle of high size variability in males and provided compelling evidence that large males have a significant reproductive advantage when male density is high and that this advantage is diminished when male density is low (48). Here, we use our model to study the effects of local male density on large-male competitive advantage.

The essential goals of a male in a female's web are to reach the female's hub and retain its hub position until copulation (24, 29 ). The competitive advantage of a male in a multimale setup can, therefore, be quantified in terms of how often it reaches the hub first ("first arrival") and how effective it is at retaining its hub position ("hub retainment"). To study how local male density affects large-male advantage in terms of these two measures, we simulated male competition in low-density and high-density setups, as illustrated in Fig. $4 A$, Inset and $C$, Inset. The number of simulated males in the high-density simulations (seven and eight males) is motivated by observations in the wild (48) and by our simulations of high competitive pressures in the proximity of the web, which indicate that a higher density of males in a single female's web is very unstable (SI Appendix, Fig. S11).

First, we explore the outcomes of competition with respect to first arrival (Fig. $4 A$ and $B$ ). We simulated the competition between two probed males that enter the female's web at the same time and at spatially equivalent positions (marked by black asterisks in Fig. $4 \mathrm{~A}$, Lower Insets). In the low-density setup, the system only includes these two competing males. In the high-density setup, five more males of the reference size are added. The additional males were initialized in a symmetric configuration (marked by blue asterisks), such that the initial positions of the probed males remained equivalent. We note that the symmetry of the initial configurations quickly disappears due to stochastic effects and is only used to ensure that there is no bias in the simulations. Of the two probed males, one is of the reference size (male 1, with $m_{1}=m_{\text {ref }}$ ), and the other is of a variable size (male 2, with $m_{2} \geq m_{1}$ ). We increased the size of male 2 in small increments, starting from the reference size and increasing to up to three times the reference size.

In Fig. $4 A$, we plot the relative probability of the larger male 2 (relative to the reference male 1) arriving first at the female's hub as a function of the size ratio between the two males. We find that the first arrival probability of the larger male increases with its size. This follows from the faster speed of the larger male and its ability to chase the smaller competitor away if they happen to encounter each other. However, even for a large size ratio of three, the smaller probed male still has a first arrival probability of $\sim 30 \%$ in the low-density setup. In the high-density setup, we find that the relative first arrival probability of the larger male is enhanced compared with the low-density case, in agreement with empirical findings (48).

When male density is high, males frequently encounter rivals and interact with them. We demonstrate how the abundance of male-male interactions creates a competitive environment that is particularly advantageous for large males. For this analysis, two males $i, j$ are considered interacting if $d_{i j}$ is within the range of spring-like effective attraction $\left(d_{i j}<90 \mathrm{~mm}\right)$ (Fig. $\left.3 A\right)$. In Fig. $4 B$, we plot the mean time spent in male-male interactions by each of the probed males of Fig. $4 A$ during their journey from the edge of the female's web and until they reach the hub. In the low-density setup, we find that this interaction time is similar for both males and that it decreases with the size of the larger male. This shows that the stronger vibration-based repulsion that larger males exert helps them avoid long-lasting short-range interactions, as they typically chase smaller males away. The ability to avoid short-range interactions and in particular, the ultimate escalation to grappling contests becomes significant in the high-density setup. We find that increased interactions with the additional males delay the reference-sized male 1 substantially, while the larger male 2 remains effective at avoiding these interactions (SI Appendix, Fig. S12 visualizes this phenomenon in spatial interaction maps).

Finally, we study the competitive advantage of large males with respect to hub retainment. We initialized the simulations with the larger male 2 already in the female's hub (the global minimum of $\left.V_{\mathrm{fem}}\right)$ and the remaining reference-sized males entering the web in a symmetric configuration (Fig. 4 C, Insets). In Fig. $4 C$,
A

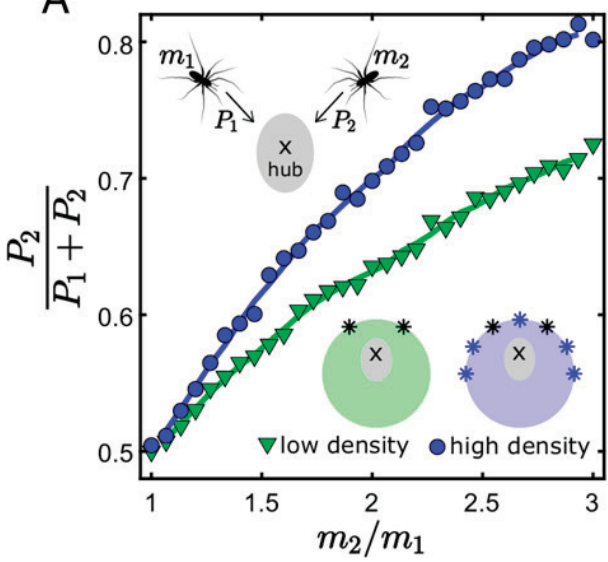

B

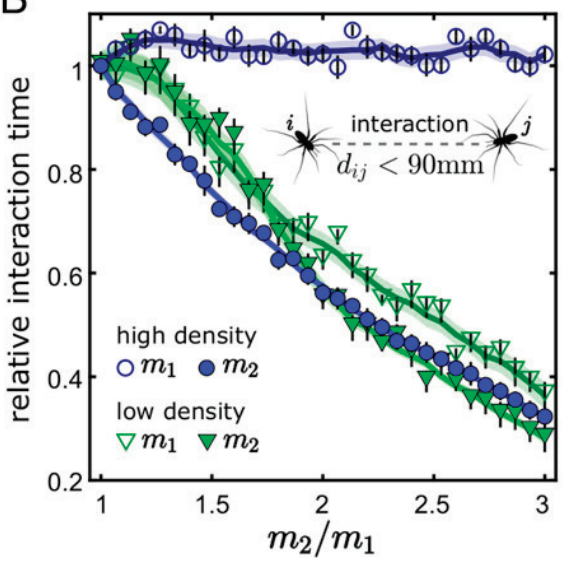

C

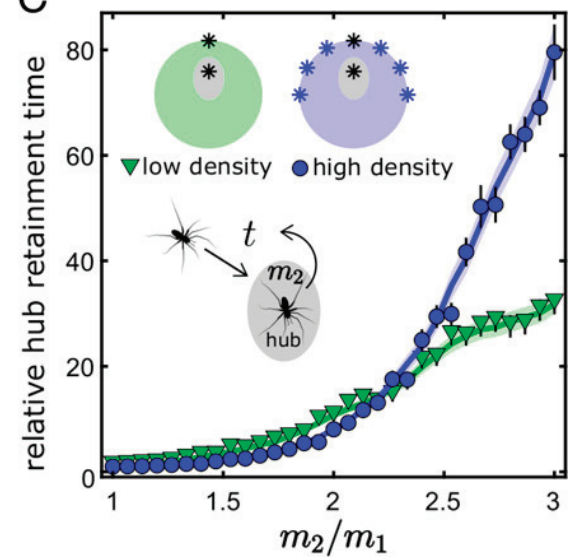

Fig. 4. Competitive benefits of large males. Model predictions-effects of male density on large-male advantage in simulations. The two probed males, with $m_{1}=m_{\text {ref }}$ and $m_{2} \geq m_{1}$, were initialized as denoted by the black asterisks. The additional males in the high-density setup, with $m_{i}=m_{\text {ref }}$, were initialized as denoted by the blue asterisks. $(A)$ Relative first arrival probability of the larger male $2(N=2,000)$. The two probed males have respective first arrival probabilities $P_{1}$ and $P_{2}$. (B) Relative interaction time until arrival to the hub $(N=2,000)$. Two males $i, j$ are considered interacting if $d_{i j}<90$ mm (within the range of spring-like effective attraction) (Fig. $3 A$ ). Low- and high-density setups as in $A$. (C) Relative hub retainment time of the larger male $2(N=200)$. The lines are five-points moving averages. Error bars show SEM. In $B$ and $C$, each curve is normalized by the respective symmetric case (all $m_{i}=m_{\text {ref }}$ ). The Insets illustrate the initial positions of the simulated males (in $A$ and $C$ ) and the measure of interest (in $A-C$ ) for each panel. 
we plot the mean time until male 2 has lost its hub position and been replaced by any of the other males as a function of the size ratio. This time is normalized by its value in the symmetric case ( $m_{1}=m_{2}=m_{\text {ref }}$ in the respective setup). We find that the hub retainment of the larger male increases dramatically with its size. For a size ratio of three in the low-density setup, the mean hub retainment time of the larger male is $\sim 30$ times longer than that of a reference-sized male. In the high-density setup, this relative advantage is greatly pronounced. This shows that large males remain effective at retaining their hub position in high male densities, while the abundance of male-male interactions penalize small males even if they managed to obtain the hub position. Note that even large males have a finite hub retainment time, which saturates in the low-density case due to the random component of the agent's motion.

\section{Discussion}

Here, we formulated a physics-based model that describes the spatiotemporal dynamics of contestants in a natural interaction arena. Motivated by empirical results from the relatively simple spider system, our theoretical approach and the effective physical rules on which it is based are broadly applicable to other systems in which agents compete over a localized resource. The model treats the contestants as active Brownian particles responding only to effective physical forces and assumes minimal cognition and no memory. Despite the relative simplicity of these agents and of the effective physics that they obey, there is strong quantitative agreement between the dynamical characteristics of the model's contestant particles and the movement and interactions of real male spiders. Our results demonstrate that a minimal behavioral repertoire of responding to vibratory and geometric information is sufficient for these males to resolve the tasks that they face in the competitive web arena.

We show that self-assessment as a means of resolving contests can be interpreted in terms of variations in the effective forces between the contestants, such that assessment asymmetry maps to asymmetry in the effective forces experienced by each contestant in the various stages of contest escalation. This approach allows us to study contests between contestants of different sizes and explore how the dynamics of these contests are affected by the size asymmetry. It further allows us to explore the relationship between contest duration and the sizes of the contestants, which is widely used to characterize animal contests $(12,13)$.

Apart from allowing a quantitative comparison with the directly observable elements of contest behavior, the ability to explore the motion of contestants during contests enables predictions that are inaccessible to the classical game-theoretic models. For example, our model captures dynamical differences between the trajectories of symmetric and asymmetric contests, where the latter are increasingly characterized by directional chases as the size difference between the contestants increases. The identification of these spatiotemporal properties emphasizes the types of empirical data to be gathered in future studies of contest behavior.

Based solely on pairwise male-male interactions through effective (and nonreciprocal) interaction potentials, our model is able to capture the dynamics of competition between many contestants. In our studied system, we provide a mechanistic explanation for the previously reported relationship between large-male advantage and male density (48). We explored this phenomenon in simulations with respect to two measures of contestant performance-first arrival at the resource and resource retainment. For both of these measures, we have shown that the competitive advantage of large males at high male density stems from their ability to avoid long-lasting short-range interactions. The effective potentials of larger males enable them to resolve interactions with smaller rivals at a distance, while small males form long-lasting contests with other small males and therefore, suffer disproportionately from a high density of rivals. This prediction remains to be explored in future experiments but suggests that decisions by smaller males to move to webs of less attractive females $(25,56)$, which tend to contain a lower number of males, may result from the effective physics of intermale interactions rather than cognitive assessment of risk and reward. Moreover, the relationship between large-male advantage and the local density of contestants has been described in other systems (39, 57-59), suggesting that these effects are more general.

Our model establishes a framework for describing contest dynamics in terms of effective pairwise potentials, which include both repulsive and attractive terms. We showed that longestablished contest assessment strategies $(1,13,60)$, which are motivated by observed contest outcomes, can be incorporated into a physics-based framework through variations in the effective interaction potentials of our model. This demonstrates that our theoretical approach can be generalized to describe the spatiotemporal dynamics of contest behavior regardless of the specifics of the system being explored. While the details of the effective interaction potentials will vary across species, this general approach enables quantitative comparisons with experimental trajectories and can provide mechanistic insights into the nature of contests and their outcomes across taxa.

\section{Materials and Methods}

Experimental Setup. Experiments were carried out in an experimental area in Gamboa, Panama, with female and male $T$. clavipes. We collected spiders from Barro Colorado Island, Panama $\left(9.15^{\circ} \mathrm{N}, 79.85^{\circ} \mathrm{W}\right)$ in April 2018, April 2019, and September 2019 and transferred them to an experimental area in Gamboa, Panama $\left(9.12^{\circ} \mathrm{N}, 79.70^{\circ} \mathrm{W}\right)$. We placed females on $70-\times 70-\mathrm{cm}$ timber or metal frames, where they could build their webs (SI Appendix, Fig. S1), and kept males in individual plastic containers. Females built their webs overnight, and experiments were conducted the following day with females that had built webs entirely within the frames (as in SI Appendix, Fig. S1). Webs were sprayed with water once a day, and a single cricket was fed to female spiders three times a day. One of these feeding sessions occurred immediately before recording began to reduce the risk of death by an aggressive female for experimental males.

For the behavioral experiments with a single male, we placed males at initial positions near the rim of the orb web, such that the entire set of initial positions covers all of the peripheral areas of the web-in order to gauge the dynamics of males on the entire surface of the web (SI Appendix, Fig. S2). In experiments with two males, we placed the males simultaneously in the upper half of the web (above the down-facing female) and roughly at spatially equivalent positions on either sides of the female (as in SI Appendix, Fig. S1). We recorded the male and female movement on the web against a white background sheet using a Sony A7s II. All experimental work was conducted in compliance with the Smithsonian Tropical Research Institute.

Trajectory Acquisition. Using the object detection algorithm Mask-RCNN (Region-based Convolutional Neural Network), we obtained the trajectories from the behavioral recordings by training the network on a subset of manually annotated video screenshots to accurately detect individual spiders (details are in ref. 61). This resulted in a pixel mask for each detected spider, where the mask's centroid was computed as the individuals' position at that frame. These positions were then used to automatically reconstruct continuous spider trajectories using a distance-based identity assignment approach. Lastly, we used TrackUtil for manual track corrections, such as keeping a unique identity for each individual. We converted the raw tracks to the same coordinate system by shifting and scaling (from pixels to centimeters) according to the known (and consistent) dimensions of the frame supporting each female's web.

ACKNOWLEDGMENTS. We thank members of the laboratories of A.J. and N.S.G. for many fruitful discussions. This work was supported by a Smithsonian Tropical Research Institute short-term fellowship (to S.F.G.R), Deutsche Forschungsgemeinschaft Cluster of Excellence 2117 "Centre for the Advanced Study of Collective Behavior" Grant 422037984 (to S.F.G.R, R.I.E, and A.J.), the Max Planck Society (S.F.G.R, R.I.E, and A.J.), Office of Naval Research Grant N00014-19-1-2556 (to D.G.), and a Minna-James-Heineman-Stiftung research grant (to A.J.). 
1. G. Arnott, R. W. Elwood, Information gathering and decision making about resource value in animal contests. Anim. Behav. 76, 529-542 (2008).

2. G. Arnott, R. W. Elwood, Fighting for shells: How private information about resource value changes hermit crab pre-fight displays and escalated fight behaviour. Proc. Biol. Sci. 274, 3011-3017 (2007).

3. K. A. Dixon, W. H. Cade, Some factors influencing male-male aggression in the field cricket gryllus integer (time of day, age, weight and sexual maturity). Anim. Behav. 34, 340-346 (1986).

4. E. R. Keeley, J. W. Grant, Visual information, resource value, and sequential assessment in convict cichlid (Cichlasoma nigrofasciatum) contests. Behav. Ecol. 4, 345-349 (1993)

5. C. D. Kelly, J. G. J. Godin, Predation risk reduces male-male sexual competition in the Trinidadian guppy (Poecilia reticulata). Behav. Ecol. Sociobiol. 51, 95-100 (2001).

6. D. J. Kemp, J. Alcock, G. R. Allen, Sequential size assessment and multicomponent decision rules mediate aerial wasp contests. Anim. Behav. 71, 279-287 (2006).

7. G. A. Parker, Assessment strategy and the evolution of fighting behaviour. J. Theor Biol. 47, 223-243 (1974)

8. R. Elwood, K. Wood, M. Gallagher, J. Dick, Probing motivational state during agonistic encounters in animals. Nature 393, 66-68 (1998).

9. M. Enquist, O. Leimar, Evolution of fighting behaviour: Decision rules and assessment of relative strength. J. Theor. Biol. 102, 387-410 (1983)

10. R. J. H. Payne, Gradually escalating fights and displays: The cumulative assessment model. Anim. Behav. 56, 651-662 (1998).

11. P. Hammerstein, G. A. Parker, The asymmetric war of attrition. J. Theor. Biol. 96, 647-682 (1982)

12. P. Taylor, R. W. Elwood, The mismeasure of animal contests. Anim. Behav. 65, 11951202 (2003).

13. G. Arnott, R. W. Elwood, Assessment of fighting ability in animal contests. Anim. Behav. 77, 991-1004 (2009)

14. A. I. Dell et al., Automated image-based tracking and its application in ecology Trends Ecol. Evol. 29, 417-428 (2014).

15. R. Gadagkar, How to design experiments in animal behaviour. Resonance 23, 1243 1257 (2018)

16. G. A. Parker, R. A. Stuart, Animal behavior as a strategy optimizer: Evolution of resource assessment strategies and optimal emigration thresholds. Am. Nat. 110, 1055-1076 (1976)

17. P. A. Verrell, Wrestling in the red-spotted newt (Notophthalmus viridescens): Resource value and contestant asymmetry determine contest duration and outcome. Anim. Behav. 34, 398-402 (1986).

18. M. S. Wells, Effects of body size and resource value on fighting behaviour in a jumping spider. Anim. Behav. 36, 321-326 (1988).

19. M. Briffa, I. C. Hardy, "Introduction to animal contests" in Animal Contests, I. C. W. Hardy, M. Briffa, Eds. (Cambridge University Press, Cambridge, United Kingdom, 2013), pp. 1-4.

20. M. Enquist, O. Leimar, T. Ljungberg, Y. Mallner, N. Segerdahl, A test of the sequential assessment game: Fighting in the cichlid fish Nannacara anomala. Anim. Behav. 40, 1-14 (1990)

21. P. Jensen, J. Yngvesson, Aggression between unacquainted pigs-Sequential assessment and effects of familiarity and weight. Appl. Anim. Behav. Sci. 58, 49-61 (1998).

22. F. Vollrath, Male body size and fitness in the web-building spider Nephila clavipes. z. Tierpsychol. 53, 61-78 (1980).

23. J. Schneider, M. Herberstein, F. C. De Crespigny, S. Ramamurthy, M. Elgar, Sperm competition and small size advantage for males of the golden orb-web spider Nephila edulis. J. Evol. Biol. 13, 939-946 (2000)

24. T. E. Christenson, K. C. Goist, Costs and benefits of male-male competition in the orb weaving spider, Nephila clavipes. Behav. Ecol. Sociobiol. 5, 87-92 (1979).

25. L. A. Jordan, H. Kokko, M. Kasumovic, Reproductive foragers: Male spiders choose mates by selecting among competitive environments. Am. Nat. 183, 638-649 (2014).

26. J. Schneider, M. Elgar, Sexual cannibalism in Nephila plumipes as a consequence of female life history strategies. J. Evol. Biol. 15, 84-91 (2002).

27. M. W. Foellmer, D. J. Fairbairn, Males under attack: Sexual cannibalism and its consequences for male morphology and behaviour in an orb-weaving spider. Evol. Ecol. Res. 6, 163-181 (2004)

28. M. Andersson, Sexual Selection (Princeton University Press, 1994), vol. 72.

29. C. W. Moore, The life cycle, habitat and variation in selected web parameters in the spider, Nephila clavipes Koch (Araneidae). Am. Midl. Nat. 98, 95-108 (1977).

30. M. Landolfa, F. Barth, Vibrations in the orb web of the spider Nephila clavipes: Cues for discrimination and orientation. J. Comp. Physiol. A Neuroethol. Sens. Neural Behav. Physiol. 179, 493-508 (1996).

31. T. A. Blackledge, M. Kuntner, I. Agnarsson, "The form and function of spider orb webs: Evolution from silk to ecosystems" in Advances in Insect Physiology, J. Casas, Ed. (Elsevier, 2011), vol. 41, pp. 175-262.
32. M. E. Herberstein, A. E. Wignall, E. A. Hebets, J. M. Schneider, Dangerous mating systems: Signal complexity, signal content and neural capacity in spiders. Neurosci. Biobehav. Rev. 46, 509-518 (2014).

33. R. K. Thomas, Lloyd Morgan's Canon in Comparative Psychology: A Handbook (Garland Publishing Co., New York, NY, 1998), pp. 156-163.

34. D. O. Fisher, A. Cockburn, The large-male advantage in brown antechinuses: Female choice, male dominance, and delayed male death. Behav. Ecol. 17, 164-171 (2006).

35. M. Pyron, Sexual size dimorphism and phylogeny in North American minnows. Biol. J. Linn. Soc. Lond. 57, 327-341 (1996).

36. P. Lindenfors, B. S. Tullberg, Phylogenetic analyses of primate size evolution: The consequences of sexual selection. Biol. J. Linn. Soc. Lond. 64, 413-447 (1998)

37. A. Loison, J. M. Gaillard, C. Pélabon, N. G. Yoccoz, What factors shape sexual size dimorphism in ungulates? Evol. Ecol. Res. 1, 611-633 (1999).

38. T. Székely, J. D. Reynolds, J. Figuerola, Sexual size dimorphism in shorebirds, gulls, and alcids: The influence of sexual and natural selection. Evolution 54, 1404-1413 (2000)

39. H. Kokko, D. J. Rankin, Lonely hearts or sex in the city? Density-dependent effects in mating systems. Philos. Trans. R. Soc. Lond. B Biol. Sci. 361, 319-334 (2006)

40. M. E. Herberstein, Spider Behaviour: Flexibility and Versatility (Cambridge University Press, 2011)

41. S. Zschokke, K. Nakata, Spider orientation and hub position in orb webs. Naturwissenschaften 97, 43-52 (2010).

42. T. Krakauer, Thermal responses of the orb-weaving spider, Nephila clavipes (Araneae: Argiopidae). Am. Midl. Nat. 88, 245-250 (1972).

43. T. E. Christenson, S. G. Brown, P. A. Wenzl, E. M. Hill, K. C. Goist, Mating behavior of the golden-orb-weaving spider, Nephila clavipes. I. Female receptivity and male courtship. J. Comp. Psychol. 99, 160 (1985).

44. S. Zschokke et al., Form and function of the orb-web. Eur. Arachnol. 19, 99 (2000).

45. C. Joslin, C. Gray, Multipole expansions in two dimensions. Mol. Phys. 50, 329-345 (1983)

46. P. Romanczuk, M. Bär, W. Ebeling, B. Lindner, L. Schimansky-Geier, Active Brownian particles. Eur. Phys. J. Spec. Top. 202, 1-162 (2012).

47. G. Volpe, S. Gigan, G. Volpe, Simulation of the active Brownian motion of a microswimmer. Am. J. Phys. 82, 659-664 (2014).

48. C. C. Rittschof, Male density affects large-male advantage in the golden silk spider, Nephila clavipes. Behav. Ecol. 21, 979-985 (2010)

49. J. Cohn, F. V. Balding, T. E. Christenson, In defense of Nephila clavipes: Postmate guarding by the male golden orb-weaving spider. J. Comp. Psychol. 102, 319 (1988).

50. J. M. Schneider, M. A. Elgar, Sexual cannibalism and sperm competition in the golden orb-web spider Nephila plumipes (Araneoidea): Female and male perspectives. Behav. Ecol. 12, 547-552 (2001).

51. G. A. Parker, D. I. Rubenstein, Role assessment, reserve strategy, and acquisition of information in asymmetric animal conflicts. Anim. Behav. 29, 221-240 (1981)

52. M. Mesterton-Gibbons, J. H. Marden, L. A. Dugatkin, On wars of attrition without assessment. J. Theor. Biol. 181, 65-83 (1996).

53. R. J. Payne, M. Pagel, Escalation and time costs in displays of endurance. J. Theor. Biol. 183, 185-193 (1996).

54. P. W. Taylor, O. Hasson, D. L. Clark, Initiation and resolution of jumping spider contests: Roles for size, proximity, and early detection of rivals. Behav. Ecol. Sociobiol. 50, 403-413 (2001)

55. A. P. Bridge, R. W. Elwood, J. T. Dick, Imperfect assessment and limited information preclude optimal strategies in male-male fights in the orb-weaving spider Metellina mengei. Proc. Biol. Sci. 267, 273-279 (2000).

56. R. Neumann, J. M. Schneider, Differential investment and size-related mating strategies facilitate extreme size variation in contesting male spiders. Anim. Behav. 101, 107-115 (2015).

57. R. J. Knell, Population density and the evolution of male aggression. J. Zool. (Lond.) 278, 83-90 (2009)

58. A. Bertin, F. Cézilly, Density-dependent influence of male characters on matelocating efficiency and pairing success in the waterlouse asellus aquaticus: An experimental study. J. Zool. (Lond.) 265, 333-338 (2005).

59. D. W. Zeh, Aggression, density, and sexual dimorphism in Chernetid Pseudoscorpions (Arachnida: Pseudoscorpionida). Evolution 41, 1072-1087 (1987).

60. K. J. Chapin, P. E. C. Peixoto, M. Briffa, Further mismeasures of animal contests: A new framework for assessment strategies. Behav. Ecol. 30, 1177-1185 (2019)

61. A. L. Rodríguez-Santiago, J. A. Arias-Aguilar, A. E. Petrilli-Barceló, R. Miranda-Luna, "A simple methodology for 2D reconstruction using a CNN model" in Mexican Conference on Pattern Recognition, K. M. Figueroa Mora et al., Eds. (Springer, 2020), pp. 98-107. 